\title{
1 Genetic screening for gynaecological cancer: where are we heading?
}

$2 \quad *$ Ranjit Manchanda, ${ }^{1,2,3}$ Ian Jacobs ${ }^{4,5,6}$

$4 \quad *$ Dr Ranjit Manchanda

$5 \quad{ }^{1}$ Consultant Gynaecological Oncologist, Department of Gynaecological Oncology, Bartshealth NHS

6 Trust, Royal London Hospital, London E1 1BB, UK

$7 \quad{ }^{2}$ Senior Lecturer, Barts Cancer Institute, Charter House Square, Queen Mary University of London,

8 London EC1M 6BQ, UK

$9{ }^{3}$ Honorary Senior Lecturer, GCRC, Women's Cancer

10 University College London, London W1T 7DN, UK

11 Fax- +44(0) 2078823888

12 E mail- r.manchanda@qmul.ac.uk

14 Prof lan Jacobs

$15 \quad{ }^{4}$ President and Vice-Chancellor

16 UNSW Australia

17 Level 1, Chancellery Building, UNSW Sydney NSW 2052

$18{ }^{5}$ Honorary Professor

19 UCL, Gower Street, London UK

$20{ }^{6}$ Honorary Professor

21 University of Manchester, Oxford Road, Manchester, UK

$22 \quad \mathrm{~T}+61(0) 293852855 \mid \mathrm{F}+61(0) 293851949$

23 i.jacobs@unsw.edu.au 
Abstract/Summary

The landscape of cancer genetics in gynaecological oncology is rapidly changing. The traditional family-history based approach has limitations and misses $>50 \%$ mutation carrier. This is now being replaced by population-based approaches. The need for changing the clinical paradigm from familyhistory based to population based BRCA1/BRCA2 testing in Ashkenazi Jews is supported by data that demonstrates population-based BRCA1/BRCA2 testing does not cause psychological harm and is cost effective. This article covers various genetic testing strategies for gynaecological cancers, including population-based approaches, panel and direct-to-consumer testing as well the need for innovative approaches to genetic counselling. Advances in genetic-testing technology and computational analytics have facilitated an integrated systems medicine approach, providing increasing potential for population-based genetic testing, risk stratification and cancer prevention. Genomic information along-with biological/computational tools will be used to deliver predictive, preventive, personalized and participatory (P4) and Precision medicine in the future.

Key Words - Population screening, genetic testing, genetic screening, BRCA1, BRCA2, cancer genes, 


\section{Introduction}

The traditional approach to genetic testing for high penetrance ovarian, breast and endometrial cancer gene mutations has involved testing affected individuals from high risk families through high risk cancer genetic clinics following intensive face to face genetic counselling. This family-history (FH) driven approach requires individuals and general practitioner's to recognise and act on a significant $\mathrm{FH}$. Mutation carriers, who are unaware of their $\mathrm{FH}$, who do not appreciate the risk/significance of their $\mathrm{FH}$, who are not proactive in seeking advice, and those who lack a strong FH (eg. from small families) get excluded from this process. It is not surprising that $\mathrm{FH}$ based prediction models are only moderately effective at predicting the presence of a BRCA1/BRCA2 mutation and have poor negative likelihood ratios for predicting their absence.[1] Their performance of these models falls further in population based cohorts when comparing BRCA1/2 carrier mutation rates to those in high risk families.[2] We[2] and others[3, 4] have shown that the FH based approach misses over half the at risk mutation carriers. Similar findings where significantly large proportion of identified mutation carriers lack a strong FH of cancer have been reported in testing of breast cancer (BC), ovarian cancer (OC) and endometrial cancer (EC) case series unselected for FH.[5-11] Furthermore, our analysis of data from London genetic testing laboratories indicates that only $12 \%$ of the identifiable BRCA1/BRCA2 carriers in the Ashkenazi Jewish (AJ) population have been identified over 10 years by the current family history based approach. Modelling of the current rates of detection in the NHS (National Health Service) indicates that it will take around 45 years to identify the carriers in the London Jewish population who are detectable on the basis of a family history, and that this will still miss half the people at risk. Identified BRCA1/2 and mismatch repair mutation carriers can opt for risk reducing salpingo-oophorectomy (RRSO) to reduce their ovarian cancer risk;[12, 13] MRI/mammography screening, risk-reducing mastectomy (RRM) [14], or chemoprevention with selective estrogen receptor modulators (SERM) to reduce their breast cancer risk;[15] preventive 
(PGD).[17] Given the effective options available for ovarian, endometrial and breast cancer risk management and prevention in these high risk women, the points above raise serious questions about the adequacy of the current FH-based approach and suggest that a move towards new approaches for risk prediction and case identification are justified. All of the limitations described above can be overcome by a population based approach to genetic testing.

\section{Principles of Population Testing for Genetic Cancer}

The original 10 principles for population screening were proposed by Wilson and Jungner in 1968.[18] The criteria proposed by the United Kingdom National Screening committee (UKNSC)[19] for 'screening for late onset genetic disorders: breast and ovarian cancer' are based on these principles. The Wilson and Jungner criteria have been modified over the years by a number of others[20-22] and adapted to genetic susceptibility for disease. Khoury et al[23] and Andermann et al[24] have presented a synthesis of emerging criteria. Table-1 summarises the published criteria into three relevant categories (a) The condition and the population, (b) the screening test and (c) the screening programme. Common and unique features of UKNSC breast and ovarian cancer[19], Khoury[23] and Andermann[24] criteria are highlighted in Table-1. Maximum overlap between the 3 criteria relate to the condition and the population. Andermann criteria do not cover issues related to the screening test per se but provide more details on requirements for programme implementation. UKNSC breast and ovarian cancer criteria do not adequately cover performance of the screening test, prevalence, acceptability, cost effectiveness and evaluation of programme implementation. Criteria by Khoury et al appear most comprehensive and overlap both UKNSC breast \& ovarian cancer and Andermann criteria.

The above published criteria do not address some key issues for population screening of cancer gene mutations . It is essential that the penetrance of the gene be well established through validated studies before being incorporated into a screening programme. Initial data on risk estimates for new 
genetic discoveries may be based on small numbers with wide confidence intervals and at times do not get confirmed in validation studies. Another important issue is understanding the impact of genetic testing on psychological health and quality of life, particularly on a population basis. While there is adequate data for high risk populations, data on this in a low-risk non-Ashkenazi Jewish population are lacking. This is needed to make an appropriate assessment balancing both risks and benefits of screening. It is important for prospective well designed implementation studies on population-based genetic testing to be undertaken prior to implementing a screening programme. Downstream management pathways should be established for at risk individuals before programme implementation. As one gene may affect more than once cancer, these should also include links to management options for other cancers at risk from one mutation, for e.g., colorectal cancer in mismatch repair mutations/ Lynch Syndrome. A population based genetic-screening programme needs to also establish and outline guidelines covering ethical and legal responsibilities such as discrimination, data protection, reporting requirements, disclosure or information sharing with family and health care providers, sample and data storage and ownership as well as licensing/patent issues that may arise. In Table- 2 we present an amalgamation of published criteria as well as some additional criteria adapted for population-based genetic testing for gynaecological cancer gene mutations. The additional criteria address some of the lacunae in previously published criteria described above.

\section{Testing in high-prevalence populations: The Ashkenazi Jewish Model}

The Ashkenazi Jewish (AJ) population has been used as a 'population model' and BRCA1/BRCA2 founder mutations as a 'disease model', to investigate the pros and cons of a population based approach for testing for high penetrance dominant cancer gene mutations. BRCA1/BRCA2 mutations are 10-20 times more common in the AJ population (1 in 40 prevalence rate) $[2,3,25,26]$ compared to the general non-AJ population. Three BRCA1/BRCA2 mutations are commonly found in the Ashkenazi Jewish population and are called founder mutations: in BRCA1 exons 1 and 20 
(6174delT(c.5946delT). In addition almost all the BRCA1/BRCA2 associated risk is explained by three founder mutations making testing easier and cheaper. We compared 'population' and 'FH based' approaches for BRCA1/BRCA2 testing in the Genetic Cancer Prediction through Population Screening (GCaPPS) randomised trial in the North London AJ community.[2] Participants were randomised to FH based (only individuals fulfilling strict family history criteria used in clinical genetics underwent genetic testing) and population based (all individuals irrespective of $\mathrm{FH}$ underwent genetic testing) testing arms. We found no difference in anxiety, depression, quality-of-life, health anxiety, distress, uncertainty and overall experience of genetic testing between FH and population-based arms. This indicates that genetic testing in a low risk population does not harm quality-of-life or psychological well-being, or cause excessive health concerns and outcomes are similar to those found in high risk populations seen in cancer genetics clinics.[27-29] Population based BRCA1/BRCA2 testing also leads to an overall reduction in anxiety, distress, and uncertainty, $[2,30,31]$ though higher levels of cancer related distress in those testing positive has been reported in a single arm study.[30] While pre-test and post-test counselling was provided to all participants in the GCaPPS study, mutation carriers identified in the Israeli and Canadian studies received only post-test counselling. Data from the as well as satisfaction with population testing amongst both men and women in the Jewish population.

A key issue of concern raised by many has been that mutation penetrance with population ascertainment may be less than the penetrance estimates obtained from families attending cancer genetics clinics, which can range from $81-88 \%$ for BC and $21-65 \%$ for OC.[5, 32-34] This has been 147 addressed by:

(a) Penetrance estimates (56-64\% for BC and $16 \%$ for OC) obtained from the population based 
(b) Published meta-analysis integrating population and cases series based data reporting risks of 43$67 \%$ for BC and $14-33 \%$ for OC.[39]

(c) More recently high penetrance estimates (40-60\% for $\mathrm{BC}$ and $53-62 \%$ for $\mathrm{OC}$ ) irrespective of $\mathrm{FH}$ obtained in a large Israeli population study which corrected for previous potential biases in estimates as well as ascertainment through female carriers.[3] These data indicate that breast/ovarian cancer penetrance for AJ BRCA1/BRCA2 carriers identified through population testing and those without a strong FH are also 'high', though as expected these estimates are a bit lower than those obtained from individuals attending cancer genetic clinics.

A health-economic evaluation is essential to balance costs and benefits in the context of setting public health policy for genetic testing for BRCA1/BRCA2 mutations. Our cost-effectiveness analysis suggests that population testing for BRCA1/BRCA2 mutations in AJ women $>30$ years reduces breast and ovarian cancer incidence by $0.34 \%$ and $0.62 \%$ and saves 0.101 more Quality adjusted life-years (QALYs) leading to 33 days gain in life-expectancy. We found population-based testing is extremely cost-effective compared to traditional FH based approach, with a discounted incremental coseffectiveness ratio (ICER) of '- $£ 2079 /$ QALY'.[40] This is well below the cost-effectiveness threshold used by NICE of $£ 20,000 / Q A L Y$.[41] The overall impact of such a strategy in the UK would be a reduction in ovarian cancer cases by 276 and breast cancer cases by 508, at a discounted cost saving of $£ 3.7$ million. A strength of this study is the extensive sensitivity analyses to explore model uncertainty. This included a deterministic sensitivity analysis in which all model parameters were varied widely at the extremes of their confidence intervals or range as well as a probabilistic sensitivity analysis in which all variables are varied simultaneously across their distributions. Despite a wide range of scenarios, both deterministic and $94 \%$ of simulations on probabilistic sensitivity analysis suggested that population-screening is highly cost-effective compared with the current FH based testing.[40] It's noteworthy that a cost-saving is obtained after implementing populationscreening among UK AJ women over 30 years old. There are not many health care interventions that 
save both lives and money! This has important implications for clinical care, population/public health, as well as providers/commissioners of health care.

Successful population based mass screening strategies for logistics, costs and acceptability are best delivered outside a hospital setting. Genetic testing in a population screening programme should also be implemented outside the hospital setting. In addition, some sections/groups of the population for reasons of confidentiality do not wish to be seen going to a hospital. We have demonstrated successful recruitment to such a program using a community/high-street based model[2] and Gabai-Kapara et al[3] have successfully undertaken testing through health screening centres/ national blood banks.

\section{Implications of the AJ Model}

There is now good evidence to show that population testing for BRCA1/BRCA2 mutations in

Ashkenazi Jews fulfils the necessary principles for population screening for genetic susceptibility of disease listed above (Table-2). Hence, there is a pressing need to change the current clinical paradigm of FH based testing for BRCA1/BRCA2 founder mutations in the Jewish population to a systematic population based approach. This has recently been advocated by us and other health professionals[40, 42] as well as charity and patient groups.[43] Such a strategy if implemented can not yet established.

Findings from the AJ model, while of direct importance for the AJ population, cannot be directly extrapolated to the rest of the general (non-Jewish) population. These may however, have 
implications and be of relevance for other populations with founder mutations[44] across the world. With the falling cost of testing, as well as rising awareness, understanding, acceptance and demand for genetic testing in society, this is becoming an increasingly important area of study and investigation. Khoury et al highlighted a framework with four phases of translational research to guide the applicability of genomic discoveries for prevention in health care,[45] and estimated that only $3 \%$ of research has been directed at downstream clinical implementation. Clearly, a lot more research is needed to assess feasibility, acceptability, impact on psychological health, cost effectiveness and applicability of such an approach in lower prevalence general populations.

\section{Testing of Population based Cancer Case Series}

UK[46] and other international guidelines[31, 47-49] recommend that BRCA1/2 testing should be offered at a $\geq 10 \%$ carrier probability/risk threshold. Recently published case series data indicate that BRCA1/BRCA2 mutations are present in 11\%-23\% of non-mucinous epithelial OC.[50-56] Identification of carriers has prognostic implications, and offers opportunities to access new treatment options like PARP inhibitors and enter novel clinical trials, $[57,58]$ as well as having implications for predictive testing and cancer prevention for family members. Hence, a number of guidelines now recommend testing for all non-mucinous epithelial $\mathrm{OC}$ as well as triple negative breast cancers, [48] and a number of centres in North America and some in Europe have adopted this practice. However, despite growing demand from patient groups and charities it is not yet uniformly available in clinical practice, including across most parts of England and Europe.

Another example of population based case series ascertainment is the identification of Lynch Syndrome (LS). 1.6-5.9\% patients with endometrial cancer (EC)[11, 59-61] and 1.8-3.7\%[62] with colorectal cancer (CRC) have mismatch repair (MMR) gene (MLH1/MSH2/MSH6/PMS2) mutations/LS. Currently Amsterdam-II[63] \& Bethesda Criteria[64] are widely used to identify LS individuals. Molecular immuno histochemistry (IHC) \& microsatellite instability (MSI) analysis for 'all' EC and CRC cases is more effective at identifying MMR carriers/LS than Amsterdam-II/Bethesda or 
modified age linked criteria alone.[62, 65-67] Reflex testing of tumour tissue is followed by pre-test counselling/ informed consent for those selected for genetic testing following IHC/MSI analysis. Such an approach would also benefit non serous epithelial OC, $20 \%$ of which are MMR deficient.[68]

Despite publication of guidelines and policy recognition, $[49,69]$ lack of funding is currently preventing harmonised implementation of the population based cancer case series approach. This is greatly compounded by limited awareness and knowledge of these issues amongst treating clinicians, pathologists, general practitioners and the population at large. Implementation also has significant implications for expansion in cancer genetics services and downstream management pathways. Nevertheless, as logistics for delivery get ironed out and awareness and acceptance increases, its applicability will increase and become widespread. This approach is here to stay and will expand to other relevant cancers and gene mutations.

\section{Panel Testing and Potential for Population based Risk Stratification}

The genomic era has heralded a rapidly changing landscape in cancer genetics. Advances in genetic testing technology with massive parallel sequencing, and big strides in computational analytics enabling synthesis of complex, large volume, cross disciplinary data has facilitated an integrated systems medicine approach, which in turn is transforming diagnostic, therapeutic and preventive healthcare strategies. In addition to the traditional high penetrance genes (e.g. BRCA1, BRCA2 and MMR genes), a number of newer intermediate/ moderate penetrance genes have been recently identified for ovarian (e.g. RAD51C, RAD51D, BRIP1),[70-72] breast (e.g. PTEN, ATM, TP53, PALB2, NBN, RAD51B, and CHEK2) and other cancers. The availability of high throughput technologies has led to multiplex panel testing becoming available in clinics. This enables testing for a number of genes leading to increased efficiency in time and costs of testing. The Office of Public Health Genomics (OPHG), Centers for Disease Control and Prevention (CDC), has described the 'ACCE' model/process for evaluating genetic tests, which incorporates four key components: analytic validity; clinical validity; clinical utility; and associated ethical, legal and social implications.[73, 74] 
Burke and Zimmerman proposed an enhanced scheme for evaluation of genetic tests with significant emphasis on 'clinical utility'.[75] Concern has been expressed at the lack of precise cancer risk estimates for a number of the genes which are part of these gene testing panels. [76] This lack of adequate clinical validation before regulatory approval or clinical implementation has been construed by some as being tantamount to technological misuse.

Large multi-centre international collaborations (e.g. Breast Cancer Action Consortium (BCAC),[77] (CIMBA),[79] Collaborative Oncological Gene-environment Study (COGS)),[80] have enabled genome wide association studies (GWAS) and large-scale genotyping efforts resulting in the discovery of numerous common genetic variants associated with cancer risk.[81, 82] Around 17 such variants have been identified for $\mathrm{OC}$ and 100 for BC.[76, 83] Each individual variant is associated with only a small increase in risk. However, the risk estimate for individuals who carry multiple risk alleles is 2-3 fold higher than those with a low polygenic load.[83] OC and BC risk prediction algorithms incorporating a polygenic risk score (PRS) based on both the known common variants and the total hypothesised polygenotype in addition to BRCA1, BRCA2 and other familial effects have been developed to improve risk prediction.[83-85] For example, the lifetime OC risk for a BRCA1/BRCA2 negative woman, with two affected first degree relatives is $>5 \%$ if she is at the top $50 \%$ of the PRS distribution. In addition, a number of lifestyle, medical and personal factors such as contraceptive pill use, tubal ligation, parity, endometriosis, subfertility, age, family-history (first degree relative(s) with OC),[85] aspirin[86] and hormone replacement therapy (HRT)[87] have been shown to be associated with OC risk. Recently the population distribution of lifetime risks of OC was quantified by risk $\geq 5 \%$.[85] Development and validation of new models for OC risk prediction and population stratification is also the subject of ongoing research in the PROMISE (Predicting Risk of Ovarian 
Malignancy Improved Screening and Early detection) programme.[88] Such an approach incorporating polygenic risk information has also been suggested for $\mathrm{BC}$, where it is estimated that $11 \%$ of the population representing $34 \%$ of cases can be identified[84] for targeted chemoprevention.[89]

Rising health care costs and ever increasing price of new cancer treatments/drug therapies in a challenging economic environment further magnify the importance of newer cost-effective preventive strategies. Development of such models provides hope for the principle of using risk stratification for the purpose of targeted primary prevention and early detection. Currently the most effective method of preventing OC is risk reducing salpingo-oophorectomy (RRSO), with a reported hazard ratio (HR) for the procedure of $0.06(\mathrm{Cl}: 0.02,0.17)$ in a low-risk population[90] and 0.21 (Cl:0.12,0.39) in high-risk BRCA1/BRCA2 carriers.[13] However, surgical prevention in current clinical practice (RRSO) is usually only available as a primary prevention strategy to high risk women (life time risk $>10 \%$ ). The precise risk threshold at which RRSO should be undertaken for OC prevention needs review in the context of evaluating and implementing a population based OC risk stratification strategy. We speculate that it is likely this will lie well below the current accepted practice of $10 \%$ risk. Although Screening for OC has not yet been shown to reduce mortality,[91] incidence screening results from the UKCTOCS study published recently indicate that screening using the risk of ovarian cancer algorithm (ROCA) doubled the number of screen-detected epithelial OC compared with a fixed Ca125 cut-off[92]. Mortality outcome results from the trial are expected to be published at the end of 2015. Should a mortality effect be demonstrated, a risk based appropriately targeted OC screening programme would become feasible. Evaluation of any population strategy needs to incorporate chemoprevention options such as use of the pill[93] and other factors like aspirin[86] being identified through pooled analyses for OC, as well as Tamoxifen for BC.[89] Although current models offer limited discrimination, they do permit identification of a higher risk sub-group, towards whom effective clinical interventions may be targeted. This can contribute 
towards reducing the burden of disease in the population. The falling cost of genetic testing coupled with sophisticated modelling and emergence of better defined cost-effective therapeutic interventions will enable implementation of such a strategy for $\mathrm{OC}$ and other cancers, including $\mathrm{BC}$ in the near future. However, further research confirming 'clinical validity' and 'clinical utility' of this approach is needed before widespread implementation of such a population screening and stratification strategy.

\section{Genetic Counselling}

Pre-test genetic counselling reduces distress, improves patients' risk perception[31] and remains part of international guidelines prior to genetic testing.[47] All participants in the GCaPPS population study received pre-test and post-test counselling. Unlike GCaPPS,[2] the Israeli[3] and Canadian[4] studies did not provide pre-test counselling but reported high satisfaction with the population testing process. 'Pre-test counselling' has not yet been directly compared to an approach of 'no pretest counselling' or only 'post-test counselling' in a randomised trial. Newer approaches like telephone counselling,[94, 95] DVD based counselling[96] have been found to be non-inferior and cost-efficient compared to standard face to face counselling. There is widespread recognition that successful implementation of case series testing requires a move away from the standard face-toface genetic counselling approach. Informed consent and pre-test counselling needs to be delivered by the non-cancer genetics professional community. Different models being explored for this purpose include mainstreaming[97] and use of dedicated trained nurse specialists co-ordinated through a regional genetics service.[98] However, data comparing outcomes of these approaches are lacking. Efficient, acceptable, and cost-effective ways of delivering information on genetic risk will be needed for the successful implementation of any population-based testing program and this area requires more research. 
Specific attention also needs to be paid to pre-test counselling and post-test counselling of results in the context of panel testing. This is more complicated given the large number of genes, some without precise risk estimates or interventions of proven clinical benefit for identified carriers. In addition uncertainty exists on how to deal with variants of uncertain significance (VUS)/ incidental findings, the identification of which will increase with the number of genes tested. Results of clinically significant mutations of sufficient risk need to be returned to participants and it is important for the possibility of incidental findings as well as plans for disclosure/non-disclosure to be discussed with participants at the outset. New approach(es) to counselling for informed consent such as a 'tiered and binned' approach are being explored.[99] Information is organised into clinically relevant 'bins' and levels ('tiers') of detail given out are dependent on an individual's needs to make an informed decision. Given the potential complexity and interpretation of results, pros and cons need to be carefully discussed with patients by experienced and well-informed health professionals.[100] Specific tools/decision aids to facilitate understanding of risk and informed consent need to be developed for panel testing and any population testing strategy. In addition, the use of adjuncts like DVDs, helplines and telephone counselling approaches are yet to be evaluated outside a single gene setting.

\section{Direct to Consumer (DTC) genetic testing}

Technological and scientific developments over the last few years have led to a number of companies offering a range of genetic testing services for common genetic variants as well as rare and high penetrance single gene disorders. These services are sold directly to consumers through avenues outside the traditional health system such as via the internet, television or other means. Driven by aggressive advertising and increasing awareness, the commercial market for this has been growing at a rapid rate. Proponents of DTC testing point to increased consumer access, consumer autonomy and empowerment as advantages. A number of professional bodies, authorities, scientists 
and individuals have highlighted concerns regarding this. These concerns relate to the quality, analytic utility, clinical utility and validity of the scientific data that forms the basis of a number of reports provided by DTC companies.[101, 102] The European Society of Human Genetics (ESHG), American Society of Clinical Oncology (ASCO) and American Society of Human Genetics (ASHG) published formal policy guidelines regarding DTC testing and advertising.[102-104] Some argue that regulation and laws cannot guarantee responsible use. However a voluntary international product quality assurance certificate along the lines of ISO could control for compliance with ethical standards, counselling, scientific validity, provide commercial advantages to DTC companies and be a better option.[105] Nevertheless, there remains widespread concern in the professional community regarding overstatement of effectiveness, minimization of risks, lack of 'informed' consent, data free-speech and patient empowerment.[109] The debate will continue.

\section{Future Perspectives}


Emergence of validated data in the not too distant future will lead to widespread clinical implementation of panel testing for genes like RAD51C, RAD51D, BRIP1, PALB2, CHEK2, ATM, etc. in women with strong FH of cancer and cancer case series. Although some have suggested that population based testing for BRCA1/BRCA2 genes could now be introduced into the general nonJewish population,[110] this is still premature as data on acceptability, clinical validity and costeffectiveness are lacking and implementation studies have not been undertaken. However, this will happen in the future once these studies are undertaken. Validated models incorporating combination(s) of a range of genetic (high, moderate and low penetrant) and epidemiologic/ environmental factors will become available for clinical implementation. As new risk variants are discovered, the performance of risk prediction models will get refined and improve. It is important for epigenomic data to also be incorporated into risk prediction models and the large data sets needed to facilitate this require developing. With the declining costs of sequencing, the use of genepanel testing, as well as whole-exome and whole-genome sequencing, will become more widespread. Large scale prospective studies of general population based testing for a panel of cancer genes/genetic variants as well as epidemiologic factors incorporated into risk prediction algorithms will need to be undertaken to evaluate clinical utility, acceptability, impact on psychological health and quality of life, uptake of preventive strategies, as well as cost-effectiveness, delivery pathways, and long term health outcomes. An initial small pilot study for $\mathrm{OC}$ is proposed to commence along these lines in 2016 within the PROMISE grant.[88]

\section{Integration into P4 Medicine and Precision Medicine}

'P4 medicine' consists of Predictive, Preventive, Personalized, and Participatory medicine.[111]

'Precision medicine' includes development of prevention and treatment strategies that take individual variability into account.[112] Systems medicine driven approaches incorporating genomic information (genomic medicine) along with appropriate biological and computational tools for data 
interpretation will be used to deliver P4 and Precision medicine in the future. This will enable introduction of individualised tailored prevention and/or treatment strategies. Integration and implementation of a population screening strategy for collecting genomic and epidemiologic information will be essential for the application of P4/Precision medicine approaches for cancer prevention and treatment. Our current health care systems are concentrated primarily on treatment of disease. They are not focused on prediction /prevention and maintaining 'wellness'. Delivery of a P4/Precision medicine approach incorporating population based testing will require a big change in focus. While precision medicine delivered treatment strategies for those with cancer are likely to remain hospital led, approaches for prediction and prevention will require a move away from hospitals and clinics to the community/high-street and/or home environment. It will involve use of new and innovative information tools, resources, devices, apps and health information systems for individuals to proactively participate in managing their health. It will also require the development of new care pathways and relationships between participating individuals and healthcare providers. Providers need to deliver predictive information as well as develop downstream management pathways for delivering effective risk-reducing clinical interventions for the at-risk population and monitoring long term health outcomes. Different solutions are likely to emerge for different countries and commercial companies offering newer DTC models with built in safeguards. In addition appropriate oversight/regulatory framework will need to be integrated into this process to maximise possible impact for population benefit. Education of the public/ consumers as well as general practitioners, genetic clinicians, gynaecologists, health care providers and stake holders involved in management of these women remains a massive challenge which also needs addressing. In January 2015, President Obama announced a precision medicine initiative with cancer as an important component within the scheme.[113] Many more such initiatives and funding streams driven innovative research studies are needed to fulfil its potential. 
- The traditional family-history based approach for genetic testing has limitations and misses $>50 \%$ mutation carriers. It is being replaced by population-based approaches for genetic testing.

- Population-based BRCA1/BRCA2 testing in Ashkenazi Jews does not cause psychological harm and identifies more people at risk, reduces breast and ovarian cancer incidence and is extremely cost effective. This supports a change in the clinical paradigm in this population.

- Population-based testing of cancer case series is becoming more widespread. However, lack of funding and awareness amongst clinicians is preventing harmonised implementation. Its successful application requires counselling with new approaches like mainstreaming, involving the non cancer genetics clinical community.

- The availability of high throughput technologies has led to multiplex panel testing becoming available in clinics. However, a number of genes being tested in these panels lack precise cancer risk estimates and uncertainty exists on how to deal with VUS and incidental findings. Pros and cons need to be carefully discussed with patients by experienced and well-informed health professionals.

- A number of newer intermediate/ moderate penetrance genes and common genetic variants have recently been identified for ovarian, breast and other cancers. Development of sophisticated risk models incorporating genomic and epidemiologic information coupled with availability of high throughput technology for genetic testing and falling costs provides opportunity for using risk stratification for the purpose of targeted primary prevention and early detection.

- There has been widespread concern in the professional community regarding overstatement of effectiveness, minimization of risks, lack of 'informed' consent, data protection issues and overselling of tests by DTC companies. The appropriateness of DTC and need for proper regulation and safe-guards remains a matter of ongoing debate. 
- In the near future, emergence of validated data will lead to widespread clinical implementation of panel testing for moderate penetrance genes like RAD51C, RAD51D, BRIP1, PALB2, CHEK2, ATM, etc. in women with strong FH of cancer and OC/BC cancer case series.

- Large scale prospective studies of general population based testing for a panel of cancer genes/genetic variants as well as epidemiologic factors incorporated into risk prediction algorithms need to be undertaken to evaluate clinical utility, acceptability, impact on psychological health/ quality of life, cost-effectiveness and long term health outcomes.

- Systems medicine driven approaches incorporating genomic information (genomic medicine) along with appropriate biological and computational tools for data interpretation will be used to deliver P4 and Precision medicine in the future. This will enable introduction of individualised tailored prevention and/or treatment strategies.

(1)

a




\section{Disclosures:}

476 RM and IJ are investigators on the GCaPPS trial on population testing for BRCA mutations, funded by

477 the cancer charity The Eve Appeal. RM declares no other conflict of interest. IJ has a financial

478 interest in Abcodia, Ltd., a company formed to develop academic and commercial development of

479 biomarkers for screening and risk prediction. IJ is a member of the board of Abcodia Ltd and a

480 Director of Women's Health Specialists Ltd.

481

482 
1. Kang HH, Williams R, Leary J, Ringland C, Kirk J, Ward R. Evaluation of models to predict brca

germline mutations. Br J Cancer 95(7), 914-920 (2006).

2. Manchanda R, Loggenberg K, Sanderson S et al. Population testing for cancer predisposing brca1/brca2 mutations in the ashkenazi-jewish community: A randomized controlled trial. J Natl Cancer Inst 107(1), 379 (2015).

3. Gabai-Kapara E, Lahad A, Kaufman B et al. Population-based screening for breast and ovarian cancer risk due to brca1 and brca2. Proc Natl Acad Sci U S A 111(39), 14205-14210 (2014).

4. Metcalfe KA, Poll A, Royer $R$ et al. Screening for founder mutations in brca1 and brca2 in unselected jewish women. J Clin Oncol 28(3), 387-391 (2010).

5. King MC, Marks JH, Mandell JB. Breast and ovarian cancer risks due to inherited mutations in brca1 and brca2. Science 302(5645), 643-646 (2003).

6. Hopper JL, Southey MC, Dite GS et al. Population-based estimate of the average age-specific cumulative risk of breast cancer for a defined set of protein-truncating mutations in brca1 and brca2. Australian breast cancer family study. Cancer Epidemiol Biomarkers Prev 8(9), 741-747 (1999).

7. Peto J, Collins N, Barfoot R et al. Prevalence of brca1 and brca2 gene mutations in patients with early-onset breast cancer. J Natl Cancer Inst 91(11), 943-949 (1999).

8. Hirsh-Yechezkel G, Chetrit A, Lubin F et al. Population attributes affecting the prevalence of brca mutation carriers in epithelial ovarian cancer cases in israel. Gynecol Oncol 89(3), 494498 (2003).

9. Moller P, Hagen Al, Apold J et al. Genetic epidemiology of brca mutations--family history detects less than $50 \%$ of the mutation carriers. Eur J Cancer 43(11), 1713-1717 (2007).

10. De Sanjose $\mathrm{S}$, Leone $\mathrm{M}$, Berez $\mathrm{V}$ et al. Prevalence of brca1 and brca2 germline mutations in young breast cancer patients: A population-based study. Int J Cancer 106(4), 588-593 (2003).

11. Hampel $\mathrm{H}$, Frankel W, Panescu J et al. Screening for lynch syndrome (hereditary nonpolyposis colorectal cancer) among endometrial cancer patients. Cancer Res 66(15), 7810-7817 (2006).

12. Finch A, Beiner $M$, Lubinski J et al. Salpingo-oophorectomy and the risk of ovarian, fallopian tube, and peritoneal cancers in women with a brca1 or brca2 mutation. Jama 296(2), 185192 (2006).

13. Rebbeck TR, Kauff ND, Domchek SM. Meta-analysis of risk reduction estimates associated with risk-reducing salpingo-oophorectomy in brca1 or brca2 mutation carriers. J Nat/ Cancer Inst 101(2), 80-87 (2009).

14. Rebbeck TR, Friebel T, Lynch HT et al. Bilateral prophylactic mastectomy reduces breast cancer risk in brca1 and brca2 mutation carriers: The prose study group. J Clin Oncol 22(6), 1055-1062 (2004).

15. Cuzick J, Sestak I, Bonanni B et al. Selective oestrogen receptor modulators in prevention of breast cancer: An updated meta-analysis of individual participant data. Lancet 381(9880), 1827-1834 (2013).

16. Schmeler KM, Lynch HT, Chen LM et al. Prophylactic surgery to reduce the risk of gynecologic cancers in the lynch syndrome. N Engl J Med 354(3), 261-269 (2006). 
17. Menon U, Harper J, Sharma A et al. Views of brca gene mutation carriers on preimplantation genetic diagnosis as a reproductive option for hereditary breast and ovarian cancer. Hum Reprod, (2007).

18. Wilson J, Jungner G. Principles and practice of screening for disease. Public Health Papers no. 34 34(34), (1968).

19. Haites N, Pharoah P, Gray J, Mackay J. Screening for late onset genetic disorders- breast and ovarian cancer. Report on Workshop, (2001).

20. Goel V. Appraising organised screening programmes for testing for genetic susceptibility to cancer. Bmj 322(7295), 1174-1178 (2001).

21. Burke W, Coughlin SS, Lee NC, Weed DL, Khoury MJ. Application of population screening principles to genetic screening for adult-onset conditions. Genet Test 5(3), 201-211 (2001).

22. Wald NJ. The definition of screening. J Med Screen 8(1), 1 (2001).

23. Khoury MJ, Mccabe LL, Mccabe ER. Population screening in the age of genomic medicine. $N$ Engl J Med 348(1), 50-58 (2003).

24. Andermann A, Blancquaert I, Beauchamp S, Dery V. Revisiting wilson and jungner in the genomic age: A review of screening criteria over the past 40 years. Bull World Health Organ 86(4), 317-319 (2008).

25. Hartge P, Struewing JP, Wacholder S, Brody LC, Tucker MA. The prevalence of common brca1 and brca2 mutations among ashkenazi jews. Am J Hum Genet 64(4), 963-970 (1999).

26. Roa BB, Boyd AA, Volcik K, Richards CS. Ashkenazi jewish population frequencies for common mutations in brca1 and brca2. Nat Genet 14(2), 185-187 (1996).

27. Nelson HD, Huffman LH, Fu R, Harris EL. Genetic risk assessment and brca mutation testing for breast and ovarian cancer susceptibility: Systematic evidence review for the u.S. Preventive services task force. Ann Intern Med 143(5), 362-379 (2005).

28. Schlich-Bakker KJ, Ten Kroode HF, Ausems MG. A literature review of the psychological impact of genetic testing on breast cancer patients. Patient Educ Couns 62(1), 13-20 (2006).

29. Sivell S, Iredale R, Gray J, Coles B. Cancer genetic risk assessment for individuals at risk of familial breast cancer. Cochrane Database Syst Rev (2), CD003721 (2007).

30. Metcalfe KA, Poll A, Llacuachaqui $\mathrm{M}$ et al. Patient satisfaction and cancer-related distress among unselected jewish women undergoing genetic testing for brca1 and brca2. Clin Genet 78(5), 411-417 (2010).

31. Nelson HD, Fu R, Goddard K et al. In: Risk assessment, genetic counseling, and genetic testing for brca-related cancer: Systematic review to update the u.S. Preventive services task force recommendation, (Ed.^(Eds). Rockville (MD) (2013).

32. Chen $\mathrm{S}$, Iversen ES, Friebel T et al. Characterization of brca1 and brca2 mutations in a large united states sample. J Clin Oncol 24(6), 863-871 (2006).

33. Satagopan JM, Boyd J, Kauff ND et al. Ovarian cancer risk in ashkenazi jewish carriers of brca1 and brca2 mutations. Clin Cancer Res 8(12), 3776-3781 (2002).

34. Evans DG, Shenton A, Woodward E, Lalloo F, Howell A, Maher ER. Penetrance estimates for brca1 and brca2 based on genetic testing in a clinical cancer genetics service setting: Risks of breast/ovarian cancer quoted should reflect the cancer burden in the family. BMC Cancer 8 , 155 (2008).

35. Chatterjee N, Kalaylioglu Z, Shih JH, Gail MH. Case-control and case-only designs with genotype and family history data: Estimating relative risk, residual familial aggregation, and cumulative risk. Biometrics 62(1), 36-48 (2006).

36. Chatterjee N, Shih J, Hartge P, Brody L, Tucker M, Wacholder S. Association and aggregation analysis using kin-cohort designs with applications to genotype and family history data from the washington ashkenazi study. Genet Epidemiol 21(2), 123-138 (2001).

37. Chatterjee N, Wacholder S. A marginal likelihood approach for estimating penetrance from kin-cohort designs. Biometrics 57(1), 245-252 (2001). 
38. Struewing JP, Hartge $P$, Wacholder $S$ et al. The risk of cancer associated with specific mutations of brca1 and brca2 among ashkenazi jews. N Engl J Med 336(20), 1401-1408 (1997).

39. Antoniou AC, Pharoah PD, Narod S et al. Breast and ovarian cancer risks to carriers of the brca1 5382insc and 185delag and brca2 6174delt mutations: A combined analysis of 22 population based studies. J Med Genet 42(7), 602-603 (2005).

40. Manchanda R, Legood R, Burnell $\mathrm{M}$ et al. Cost-effectiveness of population screening for brca mutations in ashkenazi jewish women compared with family history-based testing. J Natl Cancer Inst 107(1), 380 (2015).

41. Nice. Social value judgements: Principles for the development of nice guidance. (2008).

42. Levy-Lahad E, Lahad A, King MC. Precision medicine meets public health: Population screening for brca1 and brca2. J Natl Cancer Inst 107(1), 420 (2015).

43. Gallagher J. 'Screen more' for cancer risk genes. http://www.bbc.co.uk/news/health30246072\# (2014).

44. Ferla R, Calo V, Cascio S et al. Founder mutations in brca1 and brca2 genes. Ann Oncol 18 Suppl 6, vi93-98 (2007).

45. Khoury MJ, Gwinn M, Yoon PW, Dowling N, Moore CA, Bradley L. The continuum of translation research in genomic medicine: How can we accelerate the appropriate integration of human genome discoveries into health care and disease prevention? Genet Med 9(10), 665-674 (2007).

46. Nice. Familial breast cancer: Classification and care of people at risk of familial breast cancer and management of breast cancer and related risks in people with a family history of breast cancer. (2013).

47. American society of clinical oncology policy statement update: Genetic testing for cancer susceptibility. J Clin Oncol 21(12), 2397-2406 (2003).

48. Lancaster JM, Powell CB, Chen LM, Richardson DL. Society of gynecologic oncology statement on risk assessment for inherited gynecologic cancer predispositions. Gynecol Oncol 136(1), 3-7 (2015).

49. Sgo. Sgo clinical practice statement: Genetic testing for ovarian cancer. https://www.sgo.org/clinical-practice/guidelines/genetic-testing-for-ovarian-cancer/ (2014).

50. Integrated genomic analyses of ovarian carcinoma. Nature 474(7353), 609-615 (2011).

51. Alsop K, Fereday S, Meldrum C et al. Brca mutation frequency and patterns of treatment response in brca mutation-positive women with ovarian cancer: A report from the australian ovarian cancer study group. J Clin Oncol 30(21), 2654-2663 (2012).

52. Pal T, Permuth-Wey J, Betts JA et al. Brca1 and brca2 mutations account for a large proportion of ovarian carcinoma cases. Cancer 104(12), 2807-2816 (2005).

53. Pennington KP, Walsh T, Harrell Ml et al. Germline and somatic mutations in homologous recombination genes predict platinum response and survival in ovarian, fallopian tube, and peritoneal carcinomas. Clin Cancer Res 20(3), 764-775 (2014).

54. Walsh T, Casadei S, Lee MK et al. Mutations in 12 genes for inherited ovarian, fallopian tube, and peritoneal carcinoma identified by massively parallel sequencing. Proc Natl Acad Sci U S A 108(44), 18032-18037 (2011).

55. Zhang S, Royer R, Li S et al. Frequencies of brca1 and brca2 mutations among 1,342 unselected patients with invasive ovarian cancer. Gynecol Oncol 121(2), 353-357 (2011).

56. Song $\mathrm{H}$, Cicek MS, Dicks $\mathrm{E}$ et al. The contribution of deleterious germline mutations in brca1, brca2 and the mismatch repair genes to ovarian cancer in the population. Hum Mol Genet 23(17), 4703-4709 (2014).

57. Deeks ED. Olaparib: First global approval. Drugs 75(2), 231-240 (2015).

58. Ledermann J, Harter P, Gourley C et al. Olaparib maintenance therapy in patients with platinum-sensitive relapsed serous ovarian cancer: A preplanned retrospective analysis of outcomes by brca status in a randomised phase 2 trial. Lancet Oncol 15(8), 852-861 (2014). 
59. Batte BA, Bruegl AS, Daniels MS et al. Consequences of universal msi/ihc in screening endometrial cancer patients for lynch syndrome. Gynecol Oncol 134(2), 319-325 (2014).

60. Ferguson SE, Aronson M, Pollett A et al. Performance characteristics of screening strategies for lynch syndrome in unselected women with newly diagnosed endometrial cancer who have undergone universal germline mutation testing. Cancer 120(24), 3932-3939 (2014).

61. Moline J, Mahdi H, Yang B et al. Implementation of tumor testing for lynch syndrome in endometrial cancers at a large academic medical center. Gynecol Oncol 130(1), 121-126 (2013).

62. Vasen HF, Blanco I, Aktan-Collan K et al. Revised guidelines for the clinical management of lynch syndrome (hnpcc): Recommendations by a group of european experts. Gut 62(6), 812823 (2013).

63. Vasen HF, Watson P, Mecklin JP, Lynch HT. New clinical criteria for hereditary nonpolyposis colorectal cancer (hnpcc, lynch syndrome) proposed by the international collaborative group on hnpcc. Gastroenterology 116(6), 1453-1456 (1999).

64. Umar A, Boland CR, Terdiman JP et al. Revised bethesda guidelines for hereditary nonpolyposis colorectal cancer (lynch syndrome) and microsatellite instability. J Nat/ Cancer Inst 96(4), 261-268 (2004).

65. Acog practice bulletin no. 147: Lynch syndrome. Obstet Gynecol 124(5), 1042-1054 (2014).

66. Kwon JS, Scott JL, Gilks CB, Daniels MS, Sun CC, Lu KH. Testing women with endometrial cancer to detect lynch syndrome. J Clin Oncol 29(16), 2247-2252 (2011).

67. Mvundura M, Grosse SD, Hampel H, Palomaki GE. The cost-effectiveness of genetic testing strategies for lynch syndrome among newly diagnosed patients with colorectal cancer. Genet Med 12(2), 93-104 (2010).

68. Ketabi Z, Bartuma K, Bernstein I et al. Ovarian cancer linked to lynch syndrome typically presents as early-onset, non-serous epithelial tumors. Gynecol Oncol 121(3), 462-465 (2011).

69. Nhs England. Clinical commissioning policy: Genetic testing for brca1 and brca2 mutations. https://www.engage.england.nhs.uk/consultation/specialised-servicesconsultation/user uploads/brca-policy.pdf (2015).

70. Loveday C, Turnbull C, Ramsay E et al. Germline mutations in rad51d confer susceptibility to ovarian cancer. Nat Genet 43(9), 879-882 (2011).

71. Loveday C, Turnbull C, Ruark E et al. Germline rad51c mutations confer susceptibility to ovarian cancer. Nat Genet 44(5), 475-476; author reply 476 (2012).

72. Rafnar T, Gudbjartsson DF, Sulem P et al. Mutations in brip1 confer high risk of ovarian cancer. Nat Genet 43(11), 1104-1107 (2011).

73. Cdc. Acce model process for evaluating genetic tests. Genomic Testing, http://www.cdc.gov/genomics/gtesting/ACCE/ (2010).

74. Haddow J, Palomaki G. Acce: A model process for evaluating data on emerging genetic tests. In: Human genome epidemiology: A scientific foundation for using genetic information to improve health and prevent disease., Khoury M,Little J,Burke W (Ed.^(Eds). Oxford University Press, USA 217-233 (2003).

75. Burke W, Zimmerman R. Moving beyond acce: An expanded framework for genetic test evaluation. http://www.phgfoundation.org/file/16270/ (2007).

76. Easton DF, Pharoah PD, Antoniou AC et al. Gene-panel sequencing and the prediction of breast-cancer risk. N Engl J Med 372(23), 2243-2257 (2015).

77. Bcac. Breast cancer action consortium. http://apps.ccge.medschl.cam.ac.uk/consortia/bcac// (2015).

78. Ocac. Ovarian cancer action consortium. http://apps.ccge.medschl.cam.ac.uk/consortia/ocac/ (2015).

79. Cimba. The consortium of investigators of modifiers of brca1/2. http://apps.ccge.medschl.cam.ac.uk/consortia/cimba/ (2015). 
80. Burton H, Chowdhury S, Dent T, Hall A, Pashayan N, Pharoah P. Public health implications from cogs and potential for risk stratification and screening. Nat Genet 45(4), 349-351 (2013).

81. Kuchenbaecker KB, Ramus SJ, Tyrer J et al. Identification of six new susceptibility loci for invasive epithelial ovarian cancer. Nat Genet 47(2), 164-171 (2015).

82. Song H, Ramus SJ, Tyrer J et al. A genome-wide association study identifies a new ovarian cancer susceptibility locus on 9p22.2. Nat Genet 41(9), 996-1000 (2009).

83. Jervis $\mathrm{S}$, Song $\mathrm{H}$, Lee $\mathrm{A}$ et al. A risk prediction algorithm for ovarian cancer incorporating brca1, brca2, common alleles and other familial effects. J Med Genet, (2015).

84. Garcia-Closas M, Gunsoy NB, Chatterjee N. Combined associations of genetic and environmental risk factors: Implications for prevention of breast cancer. J Nat/ Cancer Inst 106(11), (2014).

85. Pearce CL, Stram DO, Ness RB et al. Population distribution of lifetime risk of ovarian cancer in the united states. Cancer Epidemiol Biomarkers Prev 24(4), 671-676 (2015).

86. Trabert B, Ness RB, Lo-Ciganic WH et al. Aspirin, nonaspirin nonsteroidal anti-inflammatory drug, and acetaminophen use and risk of invasive epithelial ovarian cancer: A pooled analysis in the ovarian cancer association consortium. J Nat/ Cancer Inst 106(2), djt431 (2014).

87. Beral V, Gaitskell K, Hermon C, Moser K, Reeves G, Peto R. Menopausal hormone use and ovarian cancer risk: Individual participant meta-analysis of 52 epidemiological studies. Lancet 385(9980), 1835-1842 (2015).

88. Pattison J. Promise. Report of the 2014 Review of research supported by The Eve Appeal, 9, https://www.eveappeal.org.uk/media/190995/192009-192014 research review.pdf (2014).

89. Cuzick J, Sestak I, Cawthorn S et al. Tamoxifen for prevention of breast cancer: Extended long-term follow-up of the ibis-i breast cancer prevention trial. Lancet Oncol 16(1), 67-75 (2015).

90. Parker WH, Feskanich D, Broder MS et al. Long-term mortality associated with oophorectomy compared with ovarian conservation in the nurses' health study. Obstet Gynecol 121(4), 709-716 (2013).

91. Buys SS, Partridge E, Black A et al. Effect of screening on ovarian cancer mortality: The prostate, lung, colorectal and ovarian (plco) cancer screening randomized controlled trial. Jama 305(22), 2295-2303 (2011).

92. Menon U, Ryan A, Kalsi J et al. Risk algorithm using serial biomarker measurements doubles the number of screen-detected cancers compared with a single-threshold rule in the united kingdom collaborative trial of ovarian cancer screening. J Clin Oncol, (2015).

93. Beral V, Doll R, Hermon C, Peto R, Reeves G. Ovarian cancer and oral contraceptives: Collaborative reanalysis of data from 45 epidemiological studies including 23,257 women with ovarian cancer and 87,303 controls. Lancet 371(9609), 303-314 (2008).

94. Kinney AY, Butler KM, Schwartz MD et al. Expanding access to brca1/2 genetic counseling with telephone delivery: A cluster randomized trial. J Natl Cancer Inst 106(12), (2014).

95. Schwartz MD, Valdimarsdottir HB, Peshkin BN et al. Randomized noninferiority trial of telephone versus in-person genetic counseling for hereditary breast and ovarian cancer. $J$ Clin Oncol 32(7), 618-626 (2014).

96. Manchanda $\mathrm{R}$, Loggenberg $\mathrm{K}$, Burnell $\mathrm{M}$ et al. A non-inferiority cluster randomised trial comparing dvd-based and traditional face-to-face genetic counselling in systematic population testing for brca mutations. Presented at: 3rd Joint Cancer Genetics Group Meetin and 14th International Meeting on Psychosocial Aspects of Hereditary Cancer. Manchester, UK 2015.

97. Rahman N. Mainstreaming cancer genetics programme. http://mcgprogramme.com/brcatesting/ (2015). 
98. Tischkowitz M. Genetic testing in epithelial ovarian cancer (gteoc) study. http://www.cancerresearchuk.org/about-cancer/find-a-clinical-trial/a-study-looking-genetictesting-ovarian-cancer-gteoc\#undefined (2015).

99. Bradbury AR, Patrick-Miller L, Domchek S. Multiplex genetic testing: Reconsidering utility and informed consent in the era of next-generation sequencing. Genet Med 17(2), 97-98 (2015).

100. Sgo. Sgo clinical practice statement: Next generation cancer gene panels versus gene by gene testing. https://www.sgo.org/clinical-practice/guidelines/next-generation-cancergene-panels-versus-gene-by-gene-testing/ (2014).

101. The Genomic Medicine Foundation. Direct to consumer genetic testing'- guidelines from the british society of genetic medicine on behalf of the uk genetic/genomics community. http://www.genomicmedicine.org/direct-to-consumer-genetic-testing/ (2015).

102. Statement of the eshg on direct-to-consumer genetic testing for health-related purposes. Eur J Hum Genet 18(12), 1271-1273 (2010).

103. Robson ME, Storm CD, Weitzel J, Wollins DS, Offit K. American society of clinical oncology policy statement update: Genetic and genomic testing for cancer susceptibility. J Clin Oncol 28(5), 893-901 (2010).

104. Hudson K, Javitt G, Burke W, Byers P, Ashg Social Issues Committee. Ashg statement* on direct-to-consumer genetic testing in the united states. Am J Hum Genet 81(3), 635-637 (2007).

105. Hauskeller C. Direct to consumer genetic testing. Bmj 342, d2317 (2011).

106. Zettler PJ, Sherkow JS, Greely HT. 23andme, the food and drug administration, and the future of genetic testing. JAMA internal medicine 174(4), 493-494 (2014).

107. Downing NS, Ross JS. Innovation, risk, and patient empowerment: The fda-mandated withdrawal of 23andme's personal genome service. Jama 311(8), 793-794 (2014).

108. Annas GJ, Elias S. 23andme and the fda. N Engl J Med 370(11), 985-988 (2014).

109. Green RC, Farahany NA. Regulation: The fda is overcautious on consumer genomics. Nature 505(7483), 286-287 (2014).

110. King MC, Levy-Lahad E, Lahad A. Population-based screening for brca1 and brca2: 2014 lasker award. Jama 312(11), 1091-1092 (2014).

111. Hood L, Flores M. A personal view on systems medicine and the emergence of proactive $\mathrm{p} 4$ medicine: Predictive, preventive, personalized and participatory. New biotechnology 29(6), 613-624 (2012).

112. Collins FS, Varmus H. A new initiative on precision medicine. N Engl J Med 372(9), 793-795 (2015).

113. The White House. Remarks by the president on precision medicine. https://www.whitehouse.gov/the-press-office/2015/2001/2030/remarks-presidentprecision-medicine (2015). 